\title{
New vineyard fields: Grape Juice. Selection of grapevine species, juice making, stabilization / Nouvelle filière : jus de raisin. Sélection des cépages, élaboration, stabilisation
}

\author{
Jean-Louis Escudier ${ }^{1}$, Richard Payraud ${ }^{3}$, Emilie Brienza ${ }^{3}$, Stéphanie Moreau ${ }^{3}$, Pierre Guyot ${ }^{3}$, Alain Samson ${ }^{1}$, \\ Michel Mikolajczak ${ }^{1}$, Delphine Bouissou ${ }^{1}$, Mélanie Veyret ${ }^{1}$, Soline Caillé ${ }^{2}$, Jean-Marc Souquet ${ }^{2}$, Véronique Cheynier ${ }^{2}$, \\ Emanuelle Zumstein ${ }^{1}$, Marc Heywang ${ }^{1}$, Jean-Noël Lacapère ${ }^{1}$, Jacques Rousseau ${ }^{4}$, et Hernán Ojeda ${ }^{1}$ \\ ${ }^{1}$ INRA, UE999 Pech Rouge, Gruissan, France \\ 2 INRA, UMR 1083SPO, Montpellier, France \\ ${ }^{3}$ FOULON SOPAGLY SAS, Mâcon, France \\ ${ }^{4}$ Groupe ICV-La jasse de Maurin-Lattes, France
}

\begin{abstract}
Proposed by FOULON-SOPAGLY society and INRA, a six years applied research program was developed in France to enable creation of create specific lines to elaborate high quality juice with new adapted grapevine varieties and specialized vineyards, with adapted technology. 448 vine varieties from INRA Vassal first international collection of grapevine species were evaluated to finally select 14: $2 \mathrm{~V}$. vinifera, 9 hybrids, and 3 from INRA creation (ref A. Bouquet) by hybridization. These last references, obtained from 4 or 5 back crossing between Muscadinia. rotondifolia.X. V.vinifera, have in particular a high level of resistance against mildew and oidium.These varieties have RUN 1 and RPV1. resistance genes, with several other secondary genes on one chromosome locus. This makes access to organic market easier. Agronomic criteria are studied for this selection. Among the 14 selected grapevine species, some have high level of polyphenols ( 6 times more than alicante). Others (white and red) have a high aromatic potential. Others have a low sugar content $135 \mathrm{~g} / \mathrm{l}$ to $150 \mathrm{~g} / \mathrm{l})$ and adapted sugar/ acidity ratio. -In the cellar: The absence of fermentation and alcohol requires optimization of the process for juice making. For example by increasing polyphenols extraction and colour stability by using thermo-treatment. Flash release process coupled with enzymatic soaking on grapes at $45^{\circ} \mathrm{C}$ for example, increases 2 or 3 fold polysaccharide juice content, and increases anthocyanins extraction and hence colour intensity. With this process the draining of grape marc and juice production increase. Pressure technology by on line horizontal decanter is possible. -In juice storehouse. Electro membrane process, (oenodia membrane process) with specific membranes gives possibilities to achieve two objectives on juice: - Decrease $\mathrm{pH}$ and increase acidity of juice and colour stabilization, without any additive.
\end{abstract}

Résumé. A l'initiative de la société FOULON-SOPAGLY et de l'INRA, le projet de créer un vignoble spécialisé pour le jus de raisin, à partir de variétés de vignes différentes de toutes celles actuellement retenues pour faire du vin a vu le jour. 448 variétés de la collection mondiale de vigne de l'INRA Vassal ont été évaluées, pour en proposer à l'agrément au final 14, dont 2 Vitis vinifera, 9 hybrides producteurs directs et 3 cépages issus de la création variétale de l'INRA (obtenteur A. Bouquet). Ces dernières, particulièrement tolérantes au mildiou et à l'oïdium, sont issues de 4 recroisements successifs par Vitis vinifera à partir d'un premier hybride Vitisvinifera x Muscadinia rotundifolia, porteurs des gènes de résistance RUN 1 et RPV 1 avec des gènes secondaires associés sur le même locus. Les variétés proposées sont bien adaptées pour l'élaboration de jus bio. Leurs aptitudes agronomiques sont précisées et sont étudiées par l'ICV. Parmi les variétés retenues certaines produisent des raisins très riches en polyphénols, d'autres ont un important potentiel aromatique, d'autres une acidité élevée, certaines des hauts rendements et une plus basse teneur en sucre (135 à $150 \mathrm{~g} / \mathrm{L})$ avec un ratio sucre/ acidité faible. Il s'agit aussi d'élaborer le jus à l'appui d'innovations en les adaptant. A la cave : L'absence de fermentation et d'alcool imposent des procédés optimisés d'extraction de polyphénols par thermotraitement. L'utilisation de la Flash détente en la couplant avec une étape de macération enzymatique à $45^{\circ} \mathrm{C}$ par exemple, permet une extraction équilibrée et élevée des polyphénols (tanins et anthocyanes). Ces mêmes procédés ont permis une extraction plus forte en polysaccharides (d'un facteur 2 à 3) et un meilleur asséchement du marc au pressurage. Le pressoir peut être remplacé par un décanteur horizontal à fonctionnement continu pour assécher les marcs frais. Au chai de conditionnement : Les procédés électro-membranaires, tels que définis sur les applications vin et moût de raisin (Procédés électromembranaires Société Oenodia), selon la configuration membranaire, permettent d'assurer deux objectifs maintenant validés qualitativement et technologiquement sur jus: améliorer l'acidité et baisser le $\mathrm{pH}$ des jus sans utilisation d'additifs. 


\section{Introduction}

\subsection{Pourquoi un tel projet de recherche sur jus de raisin?}

Bien qu'historique, le produit jus de raisin ne répond pas et n'a jamais répondu à l'attente d'un consommateur adulte européen : il est trop sucré et s'est donc fait largement distancer par des jus de fruits à sucrosité et ratio sucre-acide plus bas, 12 pour le jus d'orange en particulier. L'INRA et la société FOULON-SOPAGLY, leader européen des jus de raisins et coordonnateur du projet, conduisent depuis 2009 un programme pour proposer au consommateur une gamme de jus de raisins moins sucrés et plus conformes à ses attentes : projet FUI Fijus R@isol ${ }^{1}$. Le programme vise à créer intégralement une filière spécifique dédiée au jus de raisin depuis le choix de cépages spécifiques pour donner un jus plus acide et moins sucré, à ratio sucreacide (compté en acide tartrique) ramené de 20 à 18 actuellement à moins de 16. Les jus seront issus de vignes plus productives et tolérantes aux deux principales maladies de la vigne (le mildiou et l'oïdium). Les jus seront élaborés par des procédés d'extraction innovants développés récemment pour le vin : gestion de l'acidité (et de la stabilisation tartrique par électrodialyse), meilleure extraction des arômes et des polyphénols (anthocyanes et tanins). Le projet a permis à la société FOULONSOPAGLY d'être conviée aux réflexions lancées par le Ministère de l'Agriculture Français concernant les 10 priorités agro-industrielles pour les 10 ans à venir et le projet d'être retenu dans l'axe « Diversification des produits pour une viticulture durable $»$.

\section{Le cahier des charges concernant les cépages pour le jus de raisin:}

$\checkmark$ Haut rendement (supérieur à $150 \mathrm{hL} / \mathrm{ha}$ pour les cépages rouges et si possible vers $250-300 \mathrm{hL} / \mathrm{ha}$ pour les cépages blancs)

$\checkmark$ Faible taux de sucre (de l'ordre de 120-130 g/L)

$\checkmark$ Acidité supérieure à $7 \mathrm{~g} / \mathrm{L}$ (équivalent acide sulfurique), $\mathrm{pH}$ de l'ordre de 2,8 à 3,2

$\checkmark$ Haute teneur en polyphénols pour les jus rouges (Indice des polyphénols totaux supérieurs à $3 \mathrm{~g} / \mathrm{L}$ ).

$\checkmark$ Aromatique.

$\checkmark$ Tolérances aux maladies cryptogamiques.

$\checkmark$ Bonnes aptitudes agronomiques.

\footnotetext{
${ }^{1}$ FIJUS-R@isol (2009-2014), projet intégré labellisé par les deux pôles de compétitivité Q@limed (Languedoc Roussillon) et Vitagora (Bourgogne). Partenaires : société Foulon Sopagly (coordinateur), Inra (UE Pech-Rouge, UE Vassal, UMR SPO, UMR AGAP) et IFV de Montpellier, caves coopératives SAS Roquecourbe et Vignoble de la Voie d'Héraclès, ICV et IFR 100 STIC (Inserm/Université de Bourgogne. Les différents développements de ce projet sont financés à hauteur de de 2,2 millions d'euros par le FUI, les deux Régions Occitanie (ex Languedoc Roussillon) et Bourgogne, BPI et France AgriMer. La démarche a été amendée par l'organisme de tutelle France AgriMer tout au long du projet. L'accompagnement à la gestion du projet a été effectué par la Cabinet Efficient Innovation.

Les objectifs de ce programme de recherche et les résultats acquis à novembre 2015 FIJUS-R@isol ont fait l'objet de publications dans la revue des œnologues [1-4].
}

Il s'agit aussi de corriger l'image du jus de raisin jugé trop sucré par le consommateur adulte européen en l'adaptant à son goût. La reconversion des vignes en vergers à jus de raisin suppose au préalable une sélection de nouveaux cépages adaptés à la production de jus de raisin. Dans le souci d'une conduite en culture raisonnée ou en agriculture biologique, de nouvelles variétés résistantes au mildiou et à l'oïdium doivent être retenues pour certaines de leurs caractéristiques : production de gros fruits rouges et blancs, moins sucrés, taux de polyphénols élevés et riches en acides et composés aromatiques. Afin de définir les itinéraires techniques les plus appropriés, de nouvelles pratiques culturales en lien avec l'ICV (Institut coopératif du vin), une cave coopérative et quelques viticulteurs sont en cours d'évaluation. Différentes modalités de taille, de palissage et d'irrigation/ fertirrigation doivent être explorés. Une parcelle est dédiée à la conduite en culture biologique. Les régions viticoles impliquées sont en France la région Occitanie, le Beaujolais /Val de Saône, la Charente, le vignoble du Sud-Ouest...

La maîtrise de la composition et de la richesse nutritionnelle du jus de raisin est éprouvée par le contrôle de l'acidité du produit et de la quantité de polyphénols extraits des fruits.

La disparition de l'aide communautaire à l'élaboration de jus de raisin qui contraignait économiquement tous les élaborateurs à s'approvisionner via les surplus de la filière vin renforce l'intérêt économique de ce projet en Europe.

\subsection{Enjeux économiques et contexte international}

Les enquêtes récentes en France (2013) montrent que 43\% des Français consomment tous les jours un jus de fruit, essentiellement au moment du petit déjeuner. La consommation moyenne de jus de fruit atteint maintenant en France $25 \mathrm{~L} /$ habitant/ an. La moitié est du jus d'orange, $2,6 \%$ est du jus de raisin soit environ $1 \mathrm{~L} /$ habitant/ an. La marge de progression reste forte...

\subsubsection{Les enjeux économiques sont à côté d'enjeux de recherche à l'origine de la structuration de ce programme :}

Pour la production mais également pour le transformateur, les enjeux sont donc multiples :

- Pérennisation et stabilité des approvisionnements en quantité et qualité

- Optimisation du prix au travers de contrats pluriannuels (sur 10 ans) et de rendements plus élevés

- Caractérisation et identité de la gamme de jus par rapport aux références internationales.

- Réponses particulières aux demandes des distributeurs et des consommateurs.

Pour le consommateur, l'enjeu est maximum :

- Un jus de raisin, équilibré en acidité, moins sucré, plus aromatique, pauvre en résidus phytosanitaires.

- Un assemblage de trois ou quatre jus de cépages nouveaux et différents parmi les dix sélectionnés permettrait un éventail de trente ou quarante combinaisons inédites à proposer à la consommation. 


\subsubsection{Le marché actuel du jus de raisin en Europe}

Le produit jus de raisin existe sous deux formes : le pur jus et le jus reconstitué à base de concentrés (ou jus $\mathrm{ABC}$ ). Ce dernier est principalement utilisé pour les mélanges de jus de fruits.

La consommation européenne en pur jus de raisin est de 200 millions de litres. Si l'on considère le jus de raisin concentré, la consommation européenne s'élève à 100 millions de litres.

Le marché français des jus de fruits s'établit pour 2015 à 1225 millions de litres soit plus d'un milliard d'euros en valeur, le jus de raisin n'en représente que $4 \%$ en volume. Le marché des jus de fruits est en nette progression sur les purs jus de fruits, les deux principaux parfums étant l'orange et la pomme. Néanmoins, le pur jus de raisin ne bénéficie pas pleinement de cette croissance dans la mesure où il existe peu de variation de consommation en fonction des fluctuations de prix à la baisse ou à la hausse. Cependant, en 2016, le prix du jus d'orange est annoncé en hausse significative en lien avec le développement de maladies.

\subsubsection{La production de jus de raisin en Europe}

La production française de pur jus de raisin s'établit à un peu plus de 80 millions de litres élaborés majoritairement pour les jus rouges à partir de raisins de la région d'Occitanie. La moitié, soit 40 millions de litres, est conditionnée en tant que jus de raisin, le reste est intégré dans des mélanges de fruits. Plus de $50 \%$ de la production française est exportée dans toute l'Europe.

Les acteurs européens du pur jus de raisin sont principalement basés en France et Italie (Tableau 1). Ces entreprises ont des capacités de production en volume de plus d'un million d'hectolitres. Certaines entreprises, principalement italiennes, se positionnent en complément d'autres activités principales sur des marchés de masse avec de gros volumes et des prix bas. D'autres, comme FOULON SOPAGLY, se positionnent, avec les purs jus de raisin, sur des produits de milieu et haut de gamme.

Il s'agira de porter le potentiel de production audelà de 800000 hL en augmentant significativement la part d'approvisionnement français, de diminuer le rapport sucre sur acide et de le rapprocher de celui du jus de pomme. Une caractérisation à partir de marqueurs génétiques des variétés de vignes utilisées permettra de vérifier l'origine du jus parmi les premiers cépages qui seront inscrits. Les variétés retenues permettront un meilleur respect environnemental car elles sont pour l'essentiel des hybrides avec une tolérance voir une résistance aux maladies cryptogamiques.

\subsubsection{Jus de raisin et Organisation Communautaire du Marché du vin}

Dans le système de production l'élaboration de jus de raisin était historiquement liée à celle du vin. La production de raisins pour la transformation en jus ou la vinification comporte des objectifs communs en termes de couleur, caractéristiques aromatiques voire de propriétés nutritionnelles. Mais, selon la destination du raisin, il existe des oppositions marquées sur la teneur en sucre, l'acidité et le rendement.
Le statut du jus de raisin vient d'évoluer dans le cadre de la réforme de l'Organisation Communautaire du Marché du vin. Le rôle historique était de contribuer à la résorption de l'excédent viticole avec une aide communautaire à l'élaboration du jus de raisin assortie de différentes contraintes : interdiction de s'approvisionner en zones AOC ; utilisation exclusive de moûts d'origine européenne, justification de l'utilisation des moûts de raisin de cuve pour la filière jus (DCA).

A ce jour très majoritairement constituée à partir de cépages de raisin de cuve, la matière première idéale pour l'élaboration des nouveaux jus envisagés n'existe pas. La disparition, en août 2008, de l'aide communautaire a constitué une opportunité vers la mise en place d'une filière autonome et spécifiquement dédiée au jus de raisin.

Compte tenu des prix d'achat, la rentabilité suppose des rendements élevés, de $150 \mathrm{hL} / \mathrm{ha}$ pour des jus à valeur ajoutée, à plus de $300 \mathrm{hl} / \mathrm{ha}$ pour des jus de base.

Les objectifs et résultats de ce projet ont été source de plusieurs publications [1-5].

\section{De nouveaux cépages pour faire du jus de raisin : démarche de la sélection variétale}

Pendant la période 2008/2010, 448 jus issus de différentes variétés sont passés par le dispositif de présélection selon des critères physico-chimiques, sensoriels, sanitaires et productifs (rendements). Les raisins, dans leur majorité, ont été récoltés à partir de la collection ampélographique de l'Unité Expérimentale du Domaine de Vassal https://www6.montpellier.inra.fr/vassal/ (Tableau 2).

Aucune contrainte n'a été imposée à priori par rapport à l'origine génétique des cépages : Vitis vinifera, Hybrides producteurs directs (HPD) et nouvelles créations INRA résistantes aux maladies (Tableau 3 ).

Une évaluation approfondie des 448 génotypes a été réalisée en fonction des résultats des dégustations, effectuées au Plateau d'Analyses Sensorielles de l'Inra de Montpellier et du dépistage de viroses des plantes mères, effectué par l'UMT GénoVigne. Finalement 20 variétés ont été présélectionnées et sur-greffées, à partir de 2010, sur une parcelle homogène de l'Unité Expérimentale de Pech- Rouge suivi d'une plantation directe, en 2012, au domaine du Lycée Charlemagne à Carcassonne (Fig. 1). Après 4 ans d'évaluations agronomiques, technologiques et sensorielles, 14 cépages ont été proposés avec un dossier d'inscription pour fin 2016 pour certains puis 2017 pour d'autres. Une dénomination a été proposée par la société FOULON-SOPAGLY et acceptée pour la demande en cours d'inscription au catalogue viticole (Tableau 4).

\section{Les créations variétales INRA proposées à l'inscription}

Trois des variétés retenues réf G3, G4, G18 sont des créations variétales INRA issues du travail du généticien Alain Bouquet [6] qui a participé à la mise en place de ce projet Fijus R@isol. Ces trois variétés sont issues au départ de croisements Muscadinia x V.vinifera sélectionnés pour ce projet (Fig. 2). 
Tableau 1. Les fournisseurs de pur jus de raisin en Europe (source FOULON SOPAGLY).

\begin{tabular}{|c|c|c|c|}
\hline Pays & Entreprise & Production de jus de raisin hL/an & Premier produit de l'entreprise \\
\hline \multirow{2}{*}{ FRANCE } & FOULON SOPAGLY & 400000 & Pur jus de raisin \\
\cline { 2 - 4 } & & & \\
\hline \multirow{4}{*}{ ITALIE } & SAN GABRIELE & 400000 & Jus de raisin concentré \\
\cline { 2 - 4 } & & & \\
\cline { 2 - 4 } & DISTERCOOP & 100000 & Jus de raisin concentré \\
\cline { 2 - 4 } & VINORTE de raison concentré, vinaigre \\
\cline { 2 - 4 } & POLPUVA (jus bio) & 100000 & Pur jus de raisin \\
\hline \multirow{3}{*}{ ESPAGNE } & Différents acteurs & 50000 & \multirow{2}{*}{ Jus de raisin concentré, pur jus de raisin } \\
\hline
\end{tabular}

Tableau 2. Lieu de prélèvement des jus élaborés pendant la période 2008/2010. Projet FIJUS R@isol.

\begin{tabular}{|l|l|l|l|l|}
\hline \multicolumn{1}{|c|}{ Année } & 2008 & 2009 & 2010 & Total \\
\hline UE Vassal & & & & $419(227 \mathrm{R}+192 \mathrm{~B})$ \\
\hline Domaine du Chapitre & $41(52 R+29 B)$ & $202 *(139 R+63 B)$ & $136(36 R+100 B)$ & $10(6 \mathrm{R}+4 \mathrm{~B})$ \\
\hline UE Pech Rouge & $9(6 R+3 B)$ & $6(2 R+4 B)$ & 0 & $19(9 \mathrm{R}+10 \mathrm{~B})$ \\
\hline Total & $94(62 R+32 B)$ & $216(144 R+71 B)$ & $138(36 R+102 B)$ & $448(242 \mathrm{R}+206 \mathrm{~B})$ \\
\hline
\end{tabular}

$R$ : cépages rouges; $B$ : cépages blancs ;

* 228 cépages récoltés en 2009 dont 26 écartés pour des problèmes sanitaires (viroses) ou d'excès de sucres.

Tableau 3. Origine génétique des cépages présélectionnés. Projet FIJUS R@isol.

\begin{tabular}{|l|l|l|l|l|}
\hline \multicolumn{1}{|c|}{ Année } & 2008 & 2009 & 2010 & Total \\
\hline Origine & $49(28 R+21 B)$ & $42(18 R+24 B)$ & $90(22 R+68 B)$ & $181(68 R+113 B)$ \\
\hline $\begin{array}{l}\text { Hybrides producteurs } \\
\text { directs }\end{array}$ & $32(24 R+8 B)$ & $164(124 R+40 B)$ & $46(16 R+30 B)$ & $242(164 R+78 B)$ \\
\hline $\begin{array}{l}\text { Résistants (Vitis x Musca- } \\
\text { dinia) }\end{array}$ & $13(10 R+3 B)$ & $10(3 R+7 B)$ & $2(B)$ & $25(13 R+12 B)$ \\
\hline Total & $94(62 R+32 B)$ & $216(144 R+71 B)$ & $138(36 R+102 B)$ & $448(242 R+206 B)$ \\
\hline
\end{tabular}

$R$ : cépages rouges $; B$ : cépages blancs.

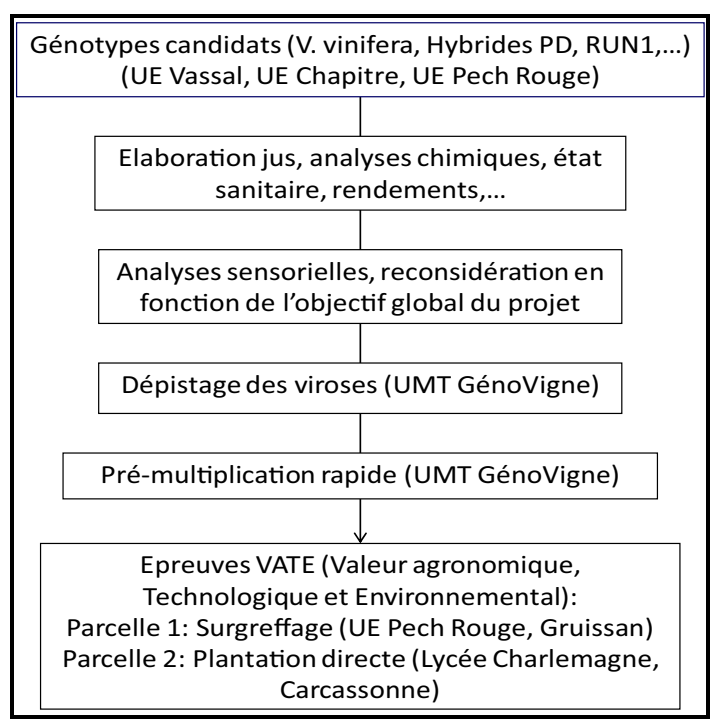

Figure 1. Représentation schématique de la démarche de la sélection variétale dans le cadre du projet Fijus R@isol.

Ces variétés ont tout de suite été repérées pour leurs grandes qualités organoleptiques, aromatiques, et leur teneur en sucre modérée. Ces variétés sont particulièrement résistantes aux maladies cryptogamiques. Cette résistance n'est pas revendiquée pour ces variétés car ceci n'a pas été l'objectif de leur sélection, mais la durabilité
Tableau 4. Dénomination proposée pour certaines des 14 cépages sélectionnés pour l'inscription au catalogue. Projet Fijus R@isol.

\begin{tabular}{|l|l|l|}
\hline & $\begin{array}{l}\text { REFERENCE } \\
\text { PROVISOIRE }\end{array}$ & $\begin{array}{l}\text { DENOMINATION } \\
\text { PROPOSEE }\end{array}$ \\
\hline 1 & Seyve-Villard 12-413 & Iloa \\
\hline 2 & Seibel 4681 & Nicora \\
\hline 3 & Galibert Coulondre 14-5 & Galibia \\
\hline 4 & Seibel 8704 & Phenolia \\
\hline 5 & Seibel 5586 & Cebriel \\
\hline 6 & Seibel 10796 & Silara \\
\hline 7 & Seibel 10096 & Farelia \\
\hline 8 & Seibel 1020 & Flot rouge \\
\hline 9 & Galibert $21-1$ & Carubis \\
\hline 10 & Caralicante & Caralicante \\
\hline 11 & Patricia INTA $\left({ }^{1}\right)$ & Patricia INTA \\
\hline 12 & G3 $\left(3197\right.$ N) $\left.{ }^{2}\right)$ & en cours \\
\hline 13 & G4 $(3184$ N $)\left({ }^{2}\right)$ & en cours \\
\hline 14 & G18 $(3199-50$ B $)\left({ }^{2}\right)$ & Recybel \\
\hline
\end{tabular}

$\left({ }^{1}\right)$ Création INTA (Argentine); $\left({ }^{2}\right)$ créations INRA.

de cette résistance est un avantage complémentaire en voie de confirmation après dix années d'expérimentations sur le vignoble de Pech Rouge. Depuis l'année 2005, ces références sur-greffées sur vinifiera (Gamay) sont observées sur une parcelle du vignoble de Pech - Rouge sur laquelle aucun traitement antifongique n'est appliqué. Les résistances à l'oïdium et au mildiou y sont vérifiées 


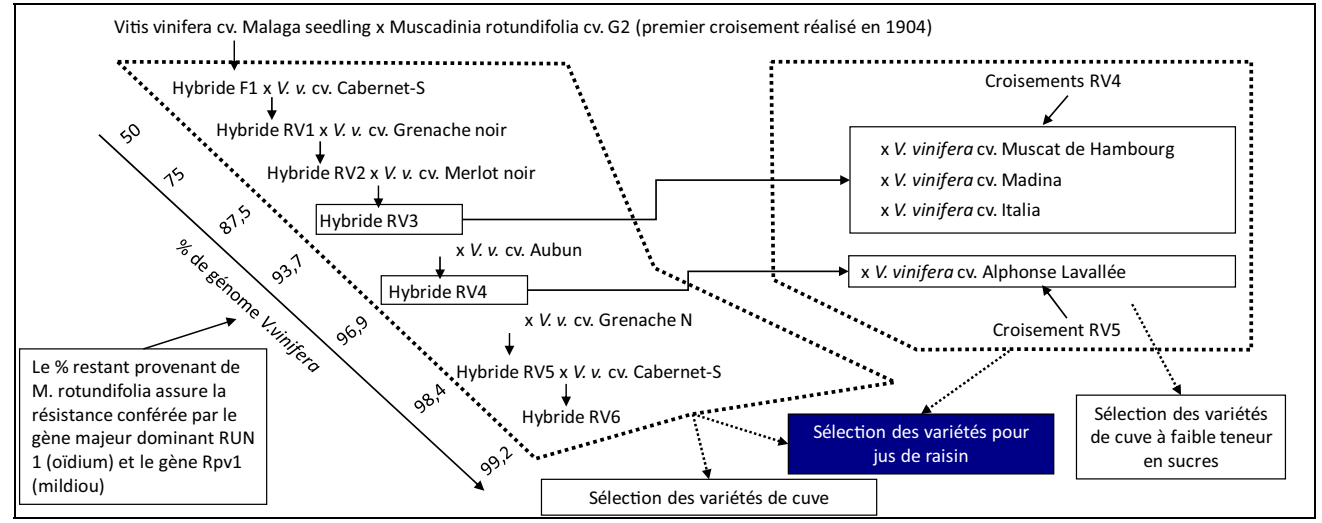

Figure 2. Sélection sur le matériel génétique tolérant à l'oïdium et au mildiou issu de recroisements inter-spécifiques Vitis vinifera $\mathrm{x}$ Muscadinia rotundifolia (obtenteur Alain Bouquet). Ojeda et al. (2010) [9].

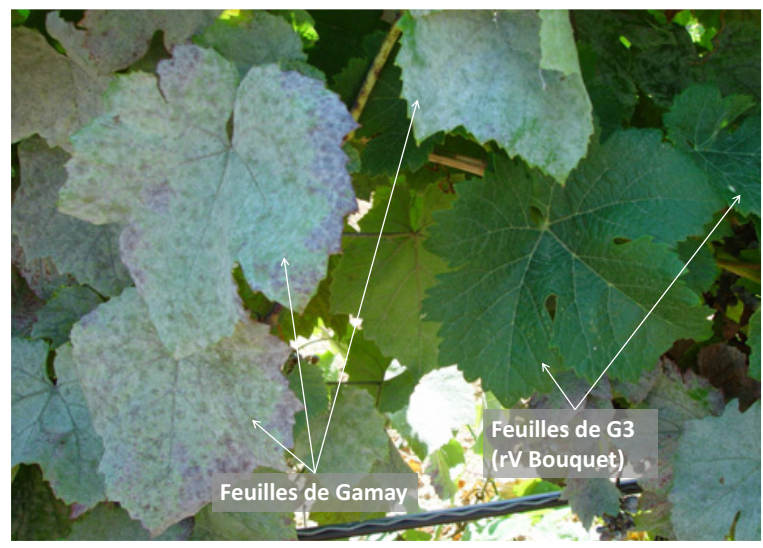

Figure 3. Parcelle de pré-sélection, sans traitements depuis 8 ans à Pech Rouge.

chaque année depuis 2005, (Fig. 3). Ces variétés sont caractérisées par la présence du gène Run 1 (Résistant à Uncinula Necator) entraînant une résistance totale à l'oïdium sur feuilles, baies et rameau, mais également par la présence du gène Rpvl (Résistant à Plasmopara viticola), entraînant une résistance partielle très élevée au mildiou [7]. Ces variétés ont une résistance à priori polygénique et durable car elles disposent de gènes secondaires de résistance en particulier RGA4 et RGA8 [8]. L'INRA, en avril 2016, a demandé leur inscription au catalogue viticole français pour leurs grandes qualités organoleptiques afin de pouvoir les inclure dans la même procédure que les autres variétés hybrides. Après inscription au catalogue viticole, elles seront déployées sous contrôle par la société FOULON-SOPAGLY et l'INRA pour en assurer un suivi dans le temps au niveau de leur durabilité de résistance aux maladies cryptogamiques.

La durabilité des résistances et l'apparition d'éventuelles maladies secondaires et ravageurs seront évaluées et contrôlées sous forme d'observations et d'éventuels traitements de protection réduit.

Caractéristiques des cépages :

Les trois cépages retenus sont issues de 4 recroisements successifs par $V$. vinifera à partir d'un premier hybride $V$. vinifera x Muscadinia rotundifolia (NC 6-15). Obtenteur: Alain Bouquet, Chercheur INRA.

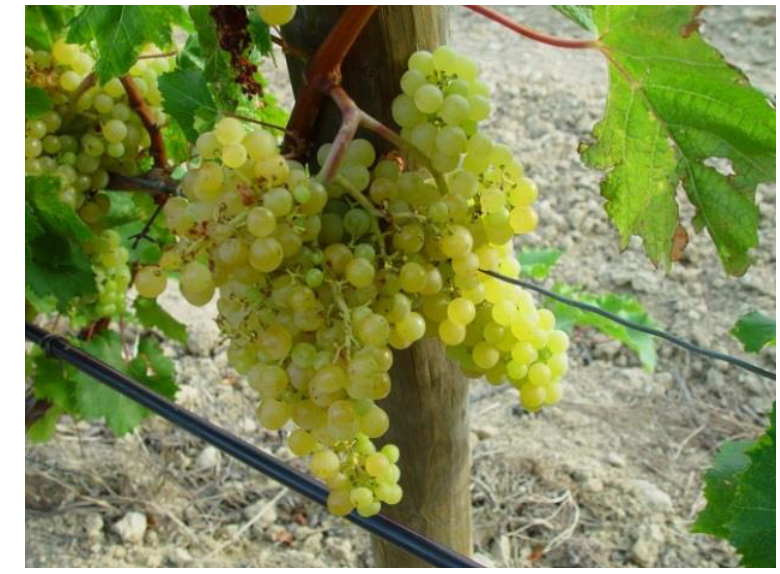

Figure 4. Cépage blanc création INRA proposée à l'inscription. Référence provisoire : G18 Dernier recroisement avec Madina.

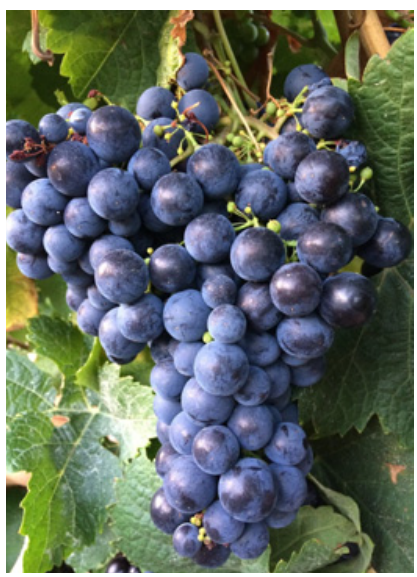

Figure 5. Cépages rouges l'inscription.

A gauche référence provisoire : G3. Dernier recroisement avec Muscat d'Hambourg.

A droite référence provisoire : G4 Dernier recroisement avec Alphonse Lavallée.

\section{Caractéristiques végétatives et productives des variétés proposées à l'inscription}

Sur les parcelles d'étude de l'INRA Pech Rouge, conduites en espalier, les variétés Iloa, Galibia, Caralicante, G 18 


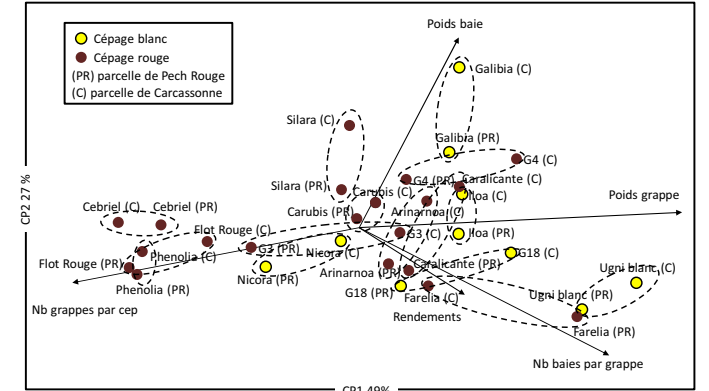

Figure 6. Analyse en composantes principales (ACP) avec les variables rendements et ses composantes des deux parcelles d'expérimentation des cépages sélectionnés pour le projet FijusR@isol. Données moyennes pour Pech Rouge (PR) et Charlemagne (C).

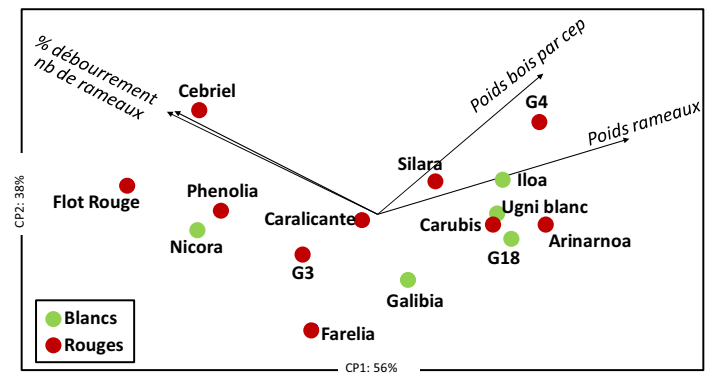

Figure 7. Analyse en composantes principales (ACP) avec les variables d'expression végétative et vigueur de la parcelle d'expérimentation des cépages sélectionnés pour le projet FijusR@isol. Données moyennes pour Pech Rouge.

ont des rendements équivalents aux deux variétés témoins (Ugni Blanc et Arinarnoa) autour de $18 \mathrm{t} / \mathrm{ha}$.

Le comportement relatif de chaque cépage vis-à-vis des rendements et de ses composantes est très stable dans les deux parcelles étudiées (Fig. 6). Flot Rouge, Cebriel et Phenolia, se différencient des autres variétés par un rendement inférieur à la moyenne malgré un grand nombre de grappes par cep. Ces trois cépages à fort potentiel phénolique (voir point 5.3) possèdent les grappes les plus petites et le plus grand nombre de grappes par rameau (données non montrées). Les plus productifs, situés à la droite de la Fig. 6, sont Iloa, Caralicante, Farelia, G18 et le témoin Ugni blanc. Les cépages Galibia, Silara, G4 et Iloa présentent les poids des baies les plus importants.

L'expression végétative, exprimée en poids de bois de taille par cep, est la plus élevée pour les références Iloa, G 18, Cebriel, Silara, Caralicante, Carubis, Ugni blanc, Arinarnoa et surtout G4 qui possède la vigueur plus élevée avec un sarment de $54 \mathrm{~g}$ contre 49 d'Iloa et 45 d'Arirnarnoa (Fig. 7).

\section{La composition analytique des jus}

L'élaboration des jus a fait l'objet d'un protocole précis adapté aux objectifs de l'étude à partir de l'ensemble des raisins récoltés. Un échantillon de $60 \mathrm{~kg}$ moyen est prélevé pour la production du jus de raisin par thermotraitement sur raisin teinturier et rouge, pressurage direct après éraflage sur raisin blanc.

Les jus de raisin ont été analysés au laboratoire de l'INRA Pech Rouge ou de l'UMR SPO à Montpellier.

Les jus retenus en comparaison des 2 jus témoins ont fait l'objet d'élaboration pendant la période 2012-2015.

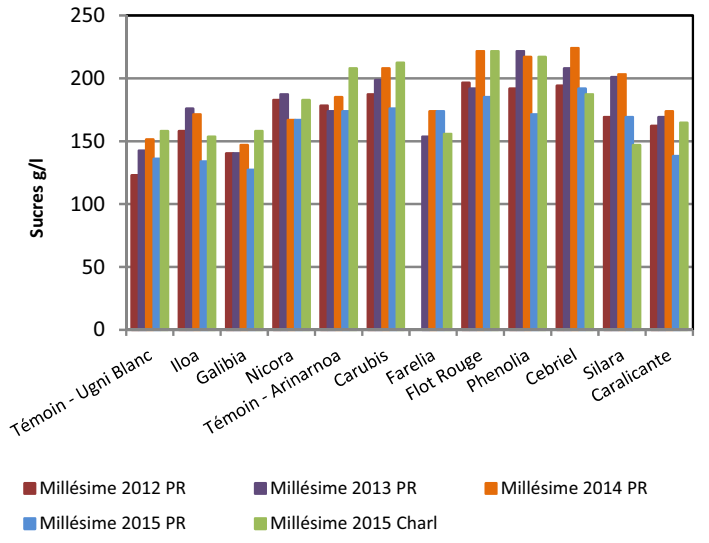

Figure 8. Teneur en sucre à la récolte des variétés proposées hors créations variétales INRA.

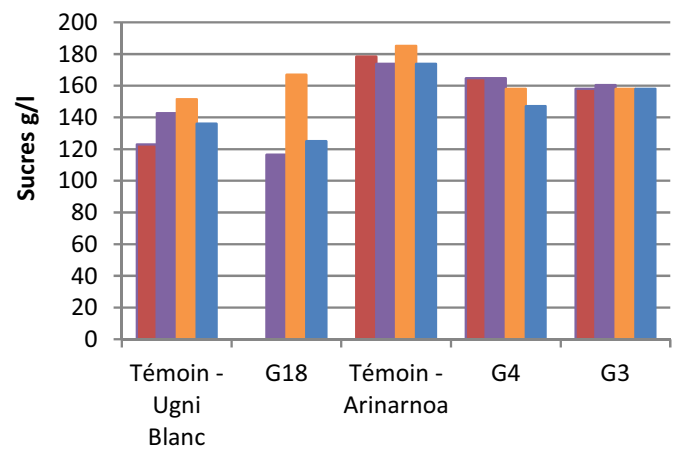

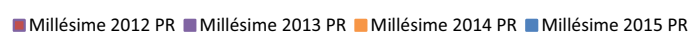

Figure 9. Teneur en sucre à la récolte des variétés créations variétales INRA (réf. G).

\subsection{Teneur en sucre des jus}

\subsubsection{Teneurs en sucre des millésimes 2012 à 2015 hors créations variétales INRA}

Les variétés Iloa, Galibia, Farelia, Silara et Caralicante ont les teneurs en sucre les plus basses inférieures à celles du témoin rouge Arinarnoa, autour d'un point moyen à $150 \mathrm{~g} / \mathrm{L}$ (Fig. 8).

\subsubsection{Teneurs en sucre des créations variétales INRA (G18, G4, G3)}

Le jus Blanc de G18 est très intéressant pour sa faible teneur en sucre, 130 à $150 \mathrm{~g} / \mathrm{L}$ selon les millésimes (Fig. 9). De même les teneurs en sucre des variétés rouges G3 et G4 sont plus faibles que celles du témoin et des autres variétés rouges retenues.

\subsection{Rapport sucre sur acide}

\subsubsection{Rapport sucre acide hors création variétale INRA}

Le ratio sucre sur acide (exprimé en acide tartrique) est le plus souvent de 15 (Fig. 10) ce qui est globalement conforme aux objectifs qui sont d'obtenir un jus moins sucré. Les jus les plus sucrés sont ceux retenus pour les critères teneur en polyphénols et couleur.

Ceci confirme qu'il est nécessaire de disposer d'une gamme de variétés pour procéder aux bons assemblages de jus. 


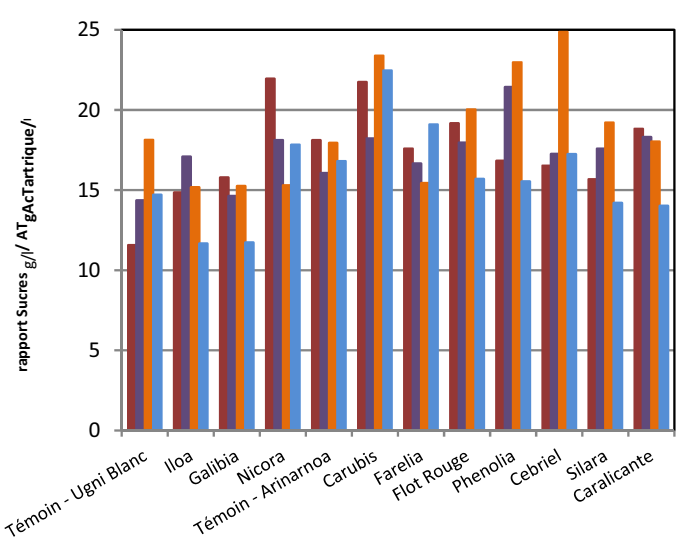

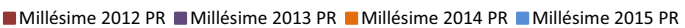

Figure 10. Rapport sucre/acide à la récolte hors créations variétales INRA.

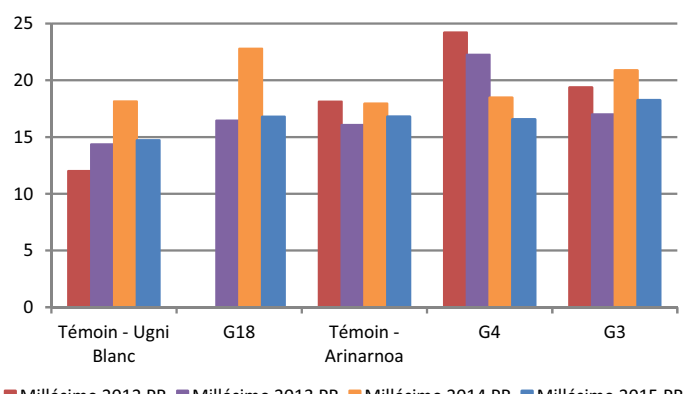

—Millésime 2012 PR Millésime 2013 PR —Millésime 2014 PR \# Millésime 2015 PR

Figure 11. Rapport Sucres (g/L) / Acidité Totale (gAcTartrique/L), à la récolte des créations variétales INRA. PR : Pech Rouge.

\subsubsection{Rapport sucre sur acide des créations variétales INRA}

Le ratio sucre/acide (exprimé en acide tartrique) se situe majoritairement entre 15 et 20 (Fig. 11). Les jus issus de la parcelle de Carcassonne se situent dans un contexte de plus haute teneur en sucre et de plus faible acidité avec de rapports sucre/acides dépassant le seuil maximal de 20, sans doute par une date de récolte trop tardive de quelques jours.

\subsection{Intensité colorante et stabilité de l'intensité colorante}

Compte tenu du haut niveau de couleur, la mesure de ce paramètre a fait l'objet d'un nouveau protocole pour les jus les plus colorés, avec une mesure sous un trajet optique de $0,2 \mathrm{~mm}$ au lieu de $1 \mathrm{~mm}$ classiquement. La bonne stabilité dans le temps (1 mois et 8 mois) a été validée pour les jus issus de chaque variété.

\subsubsection{Variétés hors créations variétales INRA}

Les jus de la parcelle référence Charlemagne ont des indices équivalents voire supérieurs à ceux de la parcelle référence Pech Rouge (Fig. 12). L'adaptation des variétés suivies dans deux contextes climatiques différents est donc bonne, y compris pour la production de jus très riches en polyphénols.

La stabilité de l'intensité colorante à 8 mois est vérifiée également. Il est intéressant de noter que la variété la plus

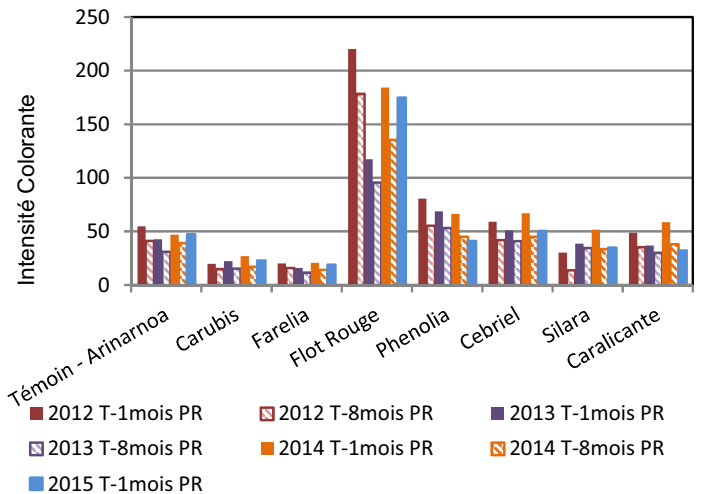

Figure 12. Suivi de l'intensité colorante des variétés rouges et teinturiers hors création variétale INRA, à 1 mois et 8 mois.

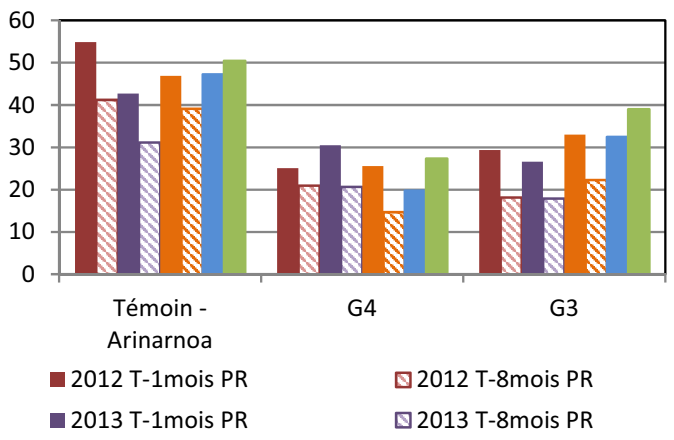

Figure 13. Suivi de l'intensité colorante des créations variétales INRA rouges, à 1 mois et 8 mois.

riche en polyphénols, Flot rouge, présente également des indices satisfaisants dans le temps au niveau stabilité de la couleur.

\subsubsection{Intensité colorante des créations variétales INRA}

Les références $\mathrm{G} 3$ et G4 ne sont pas des variétés de type teinturiers, elles ont été retenues en premier lieu pour leurs caractéristiques aromatiques. Les variétés rouges aromatiques sont très peu nombreuses. La teneur en polyphénols, l'intensité de la couleur et sa stabilité dans le temps sont tout à fait satisfaisantes (Fig. 13). Les valeurs des variétés G3 et G4 issues des deux parcelles Pech Rouge et Charlemagne sont très proches. Ceci est un élément supplémentaire très favorable.

\subsubsection{Est-il possible d'obtenir des jus colorés sans thermo traitement sur teinturier?}

Une comparaison entre thermo traitement et pressurage direct a été établie sur les variétés Flot Rouge, Phenolia, Cebriel, Silara et Caralicante.

L'intensité colorante est diminuée dans ce cas d'un facteur de 4,5 à 5 pour Flot Rouge, 13 pour Phenolia, 3 pour Cebriel, 10 pour Silara, et 4,5 pour Carilcante.

En pressurage direct c'est le jus issu de Flot Rouge qui reste le plus coloré : IC de 46,4. Ce jus reste toujours très coloré à l'œil, mais on est loin des IC de 200 à 230 obtenues par thermo traitement. La réponse à la question posée dépendra des assemblages retenus. Un jus de réserve couleur devra être travaillé au niveau du raisin par voie thermique comme présenté précédemment. 
Tableau 5. Teneur en méthanol des jus des cépages sélectionnés pour le projet FijusR@isol.

\begin{tabular}{|c|c|c|c|c|c|}
\hline Variété & $\begin{array}{c}\text { Méthanol } \\
\mathrm{mg} / \mathrm{l}\end{array}$ & Variété & $\begin{array}{c}\text { Méthanol } \\
\mathrm{mg} / \mathrm{l}\end{array}$ & Variété & $\begin{array}{c}\text { Méthanol } \\
\mathrm{mg} / \mathrm{l}\end{array}$ \\
\hline Ugni Blanc & 35,9 & Arinarnoa & 63 & Flot Rouge & 84 \\
\hline Iloa & 57,3 & Carubis & 77 & Phenolia & 57 \\
\hline Nicora & 26,8 & Farelia & 82 & Cebriel & 58 \\
\hline Galibia & 46,9 & $\mathrm{G}^{*}$ & 80 & Caralicante & 72 \\
\hline G18 & 50,9 & $\mathrm{G}^{*}$ & 81 & Silara & 82 \\
\hline
\end{tabular}

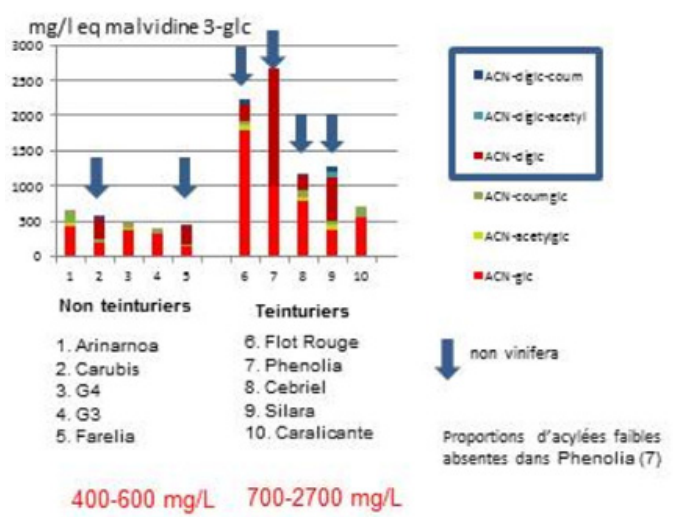

Figure 14. Composition en anthocyanes des jus issus des cépages sélectionnés pour le projet FijusR@isol.

\subsection{Teneurs en méthanol et anthocyanes diglucosides}

La teneur en méthanol a été suivie chaque année sur chaque jus issu d'hybrides et sur les créations variétales INRA.

Les teneurs en méthanol sont équivalentes à celles obtenues sur les deux Témoin et restent inférieures à $100 \mathrm{mg} / \mathrm{L}$ (Tableau 5).

Le suivi des diglucosides permet de bien séparer les jus issus d'hybrides et ceux issus de vinifera. Seuls les jus d'hybride possèdent une teneur significative en anthocyanes de type diglucoside. Ceci est d'ailleurs une méthode pour rechercher la présence de vin d'hybride dans un assemblage.

Il est intéressant de noter que les 3 créations variétales INRA (environ à 96,9\% de génome de $V$ vinifera), ne présentent pas d'anthocyanes diglucosides (Fig. 14).

\section{Technologie d'extraction des polyphénols}

La qualité des jus de raisin rouge est évaluée entre autre par leur intensité colorante. Dans cette étude, différents traitements thermiques de la vendange ont été testés afin d'extraire le maximum du potentiel de la baie, notamment des composés d'intérêt comme les polyphénols et les polysaccharides.

L'expérimentation a consisté en une comparaison de traitements biotechnologiques, par thermo macération ou par flash Détente, additionnés de préparations enzymatiques pectinolytiques spécifiques sélectionnées auprès de 3 fabricants. L'analyse chimique des jus a mis en évidence que le procédé flash Détente présente les meilleurs résultats en termes d'intensité colorante, d'indice des polyphénols totaux, de concentration en anthocyanes

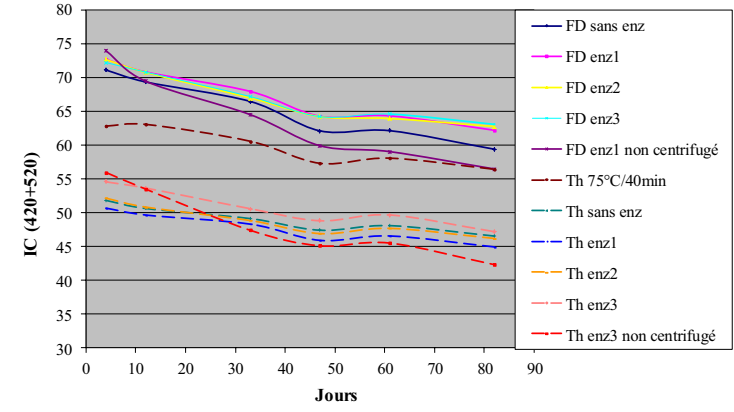

Figure 15. Suivi de l'intensité colorante sur la variété Alicante Bouschet selon les procédés étudiés : Flah détente (FD) et thermo macération à chaud $(\mathrm{Th})$. Enz $1,2,3$ : différentes enzymes pectinolytiques.

et de stabilité de cette matière colorante dans le temps. La macération pré-fermentaire à chaud dans tous les cas étudiés est le procédé le moins bien adapté en termes de gain d'extraction et de couleur. (Figure 15 sur un cas d'étude : cépage Alicante.)

En fait, les procédés de thermomacération (les plus souvent utilisés en cave pour élaborer du vin), sont dans ce cas les moins bien adaptés en termes d'extraction de couleur. L'ajout d'enzymes quant à lui impacte peu la couleur mais cela permet d'améliorer le pressurage (augmentation du rendement en jus). La clarification précoce est sur cet exemple plus favorable à une meilleure tenue de la couleur. L'extraction des polysaccharides et oligosaccharides est augmentée dans des proportions importantes pour le couplage flash détente/ macération enzymatique avant pressurage (facteur 3 par rapport au lot thermotraité). Les marcs des lots non enzymés ont des taux d'humidité plus élevés que ceux des lots enzymés ce qui montre bien que les enzymes permettent de mieux presser et donc d'obtenir de meilleurs rendements en jus. D'une manière générale les marcs de flash Détente sont mieux asséchés que les marcs de thermotraitement classiques, de $12 \%$ pour les exemples étudiés. Ceci est à mettre en relation avec la déstructuration physique des tissus végétaux lors de la mise sous vide.

L'ajout d'enzymes permet de mieux clarifier par centrifugation. Les jus de thermotraitement sont plus faciles à clarifier que les jus de flash Détente.

Les moûts ainsi traités par flash détente avec une macération enzymatique [5], pour la référence Flot Rouge en particulier ont été extraits en ligne, grâce à la force centrifuge par un décanteur horizontal selon le procédé appelé Foodec centrifuge, société Alfa Laval (11). Le taux d'assèchement atteint dans le cadre de ce procédé travaillé continu, optimisé par Alfa-Laval en lien avec INRA celui obtenu par pressoir pneumatique. Ceci permet également de pré clarifier le moût, par élimination des petites particules [10]. Sur la vendange Flot Rouge, le taux de turbidité était de 450 NTU en sortie du décanteur horizontal (950 NTU par pressoir pneumatique). Ce gain de turbidité facilite ainsi les opérations unitaires de filtration finale par membrane ou filtre sur terre. Ce procédé a initialement été développé en collaboration avec Perfect Wine, Spin off of Verona University en Italie et INRA Pech Rouge en France avec la société Vason et Linde gaz $[11,12]$. Cet outil peut intégrer un système de contrôle de l'oxydation. 
Tableau 6. Traitement des jus par électro-procédé membranaire bipolaire, impact sur le pH et la composition analytique.

\begin{tabular}{|c|c|c|c|c|c|c|c|c|c|c|c|c|}
\hline Paramètre & Unité & Incertitudes & $\begin{array}{c}\text { Jus } \\
\text { initial }\end{array}$ & $\mathrm{pH}-0,2$ & \multicolumn{2}{|r|}{ Delta } & $\mathrm{pH}-0,4$ & & Delta & $\mathrm{pH}-0,6$ & \multicolumn{2}{|r|}{ Delta } \\
\hline$p H$ & - & $\pm 0,08$ & 3,46 & 3,27 & \multicolumn{2}{|r|}{$-0,19$} & 3,07 & & $-0,39$ & 2,89 & \multicolumn{2}{|c|}{$-0,57$} \\
\hline Acidité totale & $\mathrm{gH}_{2} \mathrm{SO}_{4} \cdot \mathrm{L}^{-1}$ & $\pm 0,30$ & 4,03 & 4,47 & \multicolumn{2}{|r|}{$+0,44$} & 5,02 & & $+0,99$ & 5,49 & \multicolumn{2}{|c|}{$+1,46$} \\
\hline Abs $420 \mathrm{~nm}$ & - & $\pm 0,312$ & 4,816 & 5,026 & \multicolumn{2}{|r|}{$+0,21$} & 5,276 & & $+0,46$ & 5,541 & \multicolumn{2}{|c|}{$+0,725$} \\
\hline$A b s 520 \mathrm{~nm}$ & - & $\pm 0,634$ & 7,371 & 8,208 & \multicolumn{2}{|c|}{$+0,837$} & 9,057 & & $+1,686$ & 10,320 & \multicolumn{2}{|c|}{$+2,949$} \\
\hline$A b s 620 \mathrm{~nm}$ & - & $\pm 0,414$ & 1,812 & 1,799 & \multicolumn{2}{|c|}{$-0,013$} & 1,762 & & $-0,05$ & 1,788 & \multicolumn{2}{|c|}{$-0,024$} \\
\hline IPT DO280 & - & \pm 11 & 73,81 & 75,18 & \multicolumn{2}{|r|}{$+1,37$} & 73,76 & & $-0,05$ & 74,49 & \multicolumn{2}{|c|}{$+0,68$} \\
\hline Acide tartrique & $g \cdot L^{-1}$ & $\pm 0,47$ & 4,37 & 4,39 & \multicolumn{2}{|r|}{$+0,02$} & 4,38 & & $+0,01$ & 4,40 & \multicolumn{2}{|c|}{$+0,03$} \\
\hline Acide malique & g. $L^{-1}$ & $\pm 0,27$ & 2,96 & 3,01 & \multicolumn{2}{|r|}{$+0,05$} & 2,92 & & $-0,04$ & 3,01 & \multicolumn{2}{|c|}{$+0,05$} \\
\hline Potassium & $\mathrm{mg} \cdot \mathrm{L}^{-1}$ & \pm 90 & 1517 & 1193 & -324 & $-21,4 \%$ & 863 & -654 & $-43,1 \%$ & 550 & -967 & $-63,7 \%$ \\
\hline Sodium & $\mathrm{mg} \cdot \mathrm{L}^{-1}$ & \pm 2 & 17 & 16 & -1 & $-5,9 \%$ & 13 & -4 & $-23,5 \%$ & 10 & -7 & $-41,2 \%$ \\
\hline Magnésium & $\mathrm{mg} \cdot \mathrm{L}^{-1}$ & \pm 12 & 105 & 105 & 0 & $0,0 \%$ & 105 & 0 & $0,0 \%$ & 103 & -2 & $-1,9 \%$ \\
\hline Calcium & $\mathrm{mg} \cdot \mathrm{L}^{-1}$ & \pm 23 & 156 & 159 & +3 & $1,9 \%$ & 159 & +3 & $1,9 \%$ & 151 & -5 & $-3,2 \%$ \\
\hline
\end{tabular}

\section{Technologie d'acidification et de maitrise de $\mathrm{pH}$ et de stabilisation Tartrique}

Les travaux réalisés sur vin par l'INRA UE Pech Rouge en partenariat avec Enodia (la division œnologie d'Eurodia) à l'appui des procédés électro-membranaires ont permis d'adopter de nouvelles pratiques œnologiques maintenant bien référencées dans l'ensemble des pays vinicoles: stabilité tartrique des moûts et des vins, désacidification et acidification des moûts et des vins. Cette dernière pratique œnologique est autorisée en Europe sur vin et moût depuis 2011 (Règlement UE N53/2011). Il a été possible d'évaluer sur jus de raisin la diminution du $\mathrm{pH}$ par cette nouvelle technologie et d'atteindre ainsi un des objectifs du projet : diminuer la perception sucrée du jus de raisin.

Les membranes d'électrodialyse utilisées pour l'acidification sont de deux types : perméables aux cations (cationiques) et bipolaires. Les membranes bipolaires sont constituées d'une face anionique et d'une face cationique assemblées. Ainsi mises dans un empilement et sous l'influence d'un champ électrique, les membranes bipolaires associées à des membranes cationiques rendent possible l'exportation des seuls cations, majoritairement du potassium, et la rétention totale des anions, donc des acides organiques, et des composés non chargés. Le maintien de l'équilibre ionique acido-basique du système à la suite de l'exportation des cations se traduit par une diminution significative du $\mathrm{pH}$ [13]. Le $\mathrm{pH}$ est contrôlé en ligne pendant le traitement à l'aide d'un $\mathrm{pH}$ mètre.

Le procédé d'électrodialyse à membranes bipolaires a été testé avec un appareil pilote d'une capacité de traitement de vin maximale de $15 \mathrm{hL} / \mathrm{h}$ (Référence ED15 d'Eurodia), sur trois lots de $10 \mathrm{hL}$ de jus de raisin clarifiés de la gamme FOULON-SOPAGLY : un moût blanc muté et un jus blanc désulfité à profil similaire et un jus rouge. L'objectif était d'étudier la possibilité de réaliser 4 baisses de $\mathrm{pH}(-0,2-0,4-0,6$ et -1$)$. L'impact de ces acidifications a été évalué par des analyses physicochimiques et sensorielles [2].

L'acidification par électrodialyse au-delà de 0,6 unité $\mathrm{pH}$ est possible mais plus difficile car les capacités de traitement sont alors réduites. Les moûts mutés présentent des $\mathrm{pH}$ plus faibles, de l'ordre de $-0,5$ à $-0,7$ unité $\mathrm{pH}$ que ceux observés après désulfitage. La capacité

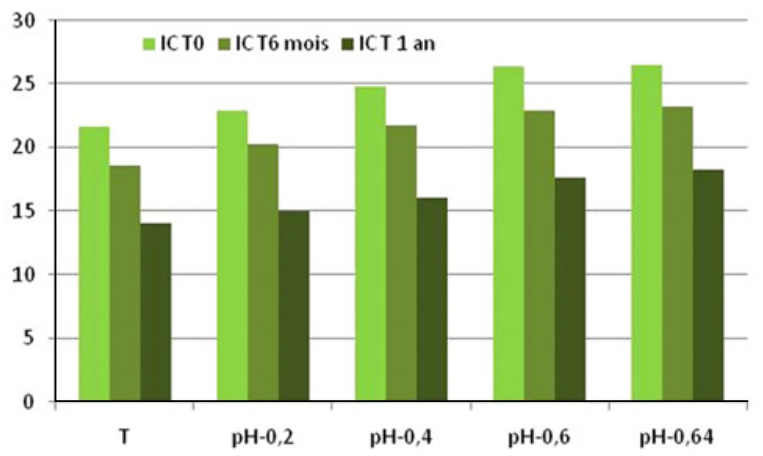

Figure 16. Evolution dans le temps de l'intensité colorante d'un jus d'Alicante Bouschet en fonction du $\mathrm{pH}$.

d'acidification de moût muté a été démontrée. La baisse de $\mathrm{pH}$ obtenue après désulfitage est inférieure de l'ordre de $-0,10$ à celle réellement effectuée sur le moût muté. L'acidification entraîne une diminution des teneurs de potassium et sodium, une augmentation des absorbances à 420 et $520 \mathrm{~nm}$ et une diminution de l'absorbance à $620 \mathrm{~nm}$ mesurée sur jus rouge (Tableau 6). Les autres paramètres ne sont pas modifiés.

A l'issue de ces essais à échelle pilote, la validation industrielle a été réalisée à partir d'une unité industrielle mobile de la société Gemstab équipée d'un empilement membranaire Modèle OENODIA ED30), sur moût muté au $\mathrm{SO}_{2}$ et sur moût désulfité.

L'analyse sensorielle réalisée sur les jus rouges et blancs montre que l'acidification entraîne une diminution de la perception sucrée sans modification de la teneur en sucre. La qualité et l'intensité aromatique sont plus élevées sur les modalités acidifiées à $-0,2$ et $-0,4$ unité $\mathrm{pH}$.

La diminution du $\mathrm{pH}$ possède un effet favorable sur la stabilité de la matière colorante. La Fig. 16 donne l'évolution de l'intensité colorante sur jus issu d'un cépage teinturier en fonction du $\mathrm{pH}$ du jus.

Une autre alternative pour diminuer la perception sucrée du jus de raisin consiste à élaborer du verjus en récoltant le raisin juste avant véraison [14]. L'assemblage de verjus $(\mathrm{pH} 2,58)$ au jus blanc $(\mathrm{pH} \mathrm{3,55)}$ utilisé pour l'expérimentation de validation à petite échelle, a nécessité $10 \%$ de verjus pour une baisse de $\mathrm{pH}$ de 0,2 unité $\mathrm{pH}$, $22 \%$ pour $-0,4$ unité $\mathrm{pH}$ et $42 \%$ pour $-0,6$ unité $\mathrm{pH}$. Cela a entrainé des augmentations des teneurs en 
potassium, sodium, acides tartrique et malique, acidité totale et absorbance à $420 \mathrm{~nm}$, ainsi qu'une diminution de la teneur en sucre.

Ces deux alternatives d'acidification, pour pouvoir être appliquées sur jus de raisin, demanderaient une adaptation réglementaire. Le procédé d'électrodialyse est autorisé sur moût pour faire du vin et non sur jus. Par ailleurs le procédé d'électrodialyse conventionnel (avec membranes anioniques et cationiques) permet d'assurer la stabilité tartrique du jus, le taux d'extraction des ions peut être adapté spécifiquement pour l'élaboration de jus concentré afin d'éviter des dépôts de tartre lors de la concentration. D'autre part pour élaborer le verjus, le jus de raisin doit être élaboré avec du raisin vert et non mûr de ce fait, ce qui demanderait une adaptation de la réglementation.

\section{Analyse sensorielle}

\subsection{Méthodologie}

Les séances d'analyses sensorielles ont été réalisées au plateau analyse sensorielle INRA Pech- Rouge/ UMR SPO. Les membres du jury comprenaient principalement l'équipe dirigeante de la société FOULON et des personnels de l'INRA UEPR, mais aussi occasionnellement des autres partenaires : IFV, ICV, France AgriMer.

Trois axes ont été retenus pour la sélection sensorielle des variétés : la couleur, l'odeur et en gustatif l'équilibre sucre/acidité. L'analyse a été réalisée chaque année à deux niveaux : un premier qui a permis une quantification pour chaque variété des descripteurs choisis et un deuxième niveau qualitatif pour établir un classement hédonique des variétés. Ces deux niveaux d'analyses ont permis chaque année d'éliminer des variétés sur des critères d'insuffisance ou d'excès sur les axes définis.

Pour chaque couleur de jus des variétés témoins ont été ajoutées pour comparaison (Ugni blanc et pour les jus blancs, et Arinarnoa pour les jus rouges).

Les variétés ont été évaluées d'une part par l'intensité de chaque caractéristique étudiée (couleur, arôme et équilibre), et d'autre part, leur évaluation qualitative, ceci pour chaque millésime étudié.

\subsection{Résultats d'analyse sensorielle. Comparaison des caractéristiques aromatiques}

Les résultats concernant la perception aromatique des jus étudiés, en particulier issus des créations variétales INRA, montrent que les variétés G3 et G4 ont une qualité de leurs arômes supérieure à l'Arinarnoa (témoin), la présence d'arômes 《 muscatés 》 issues de leurs géniteurs ne sont certainement pas étrangers à leurs bons classements (Fig. 17).

La variété G18 a une qualité de ses arômes supérieure à l'Ugni blanc (témoin), avec une intensité plus importante avec des arômes d'intensité plutôt forte et de bonne qualité (Fig. 18).

G18 produit également un jus de couleur moyenne et de qualité plutôt bonne avec un équilibre sucrosité /acidité plutôt bon ; cet équilibre est variable selon les millésimes(données non montrées).

Les créations variétales INRA sont les seules à donner des raisins rouges aromatiques. Les variétés hybrides teinturiers ressortent pour leur potentiel en couleur et

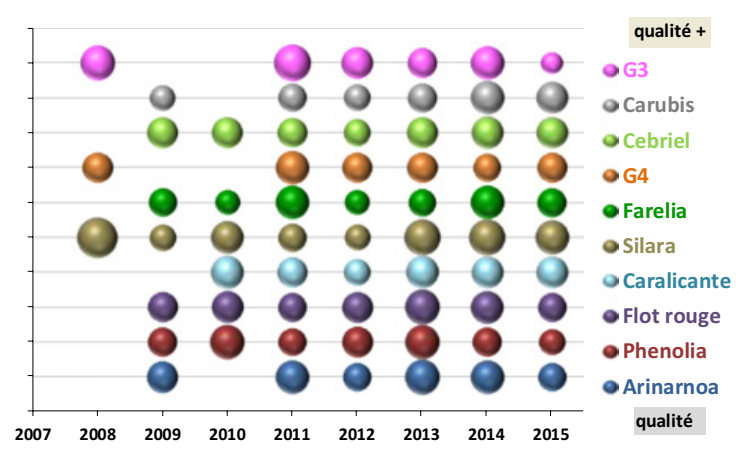

Figure 17. Evolution de la perception de l'intensité aromatique des jus rouges selon les millésimes et classification en fonction de la qualité. Grosseur de la boule en fonction de l'intensité des arômes en bouche.

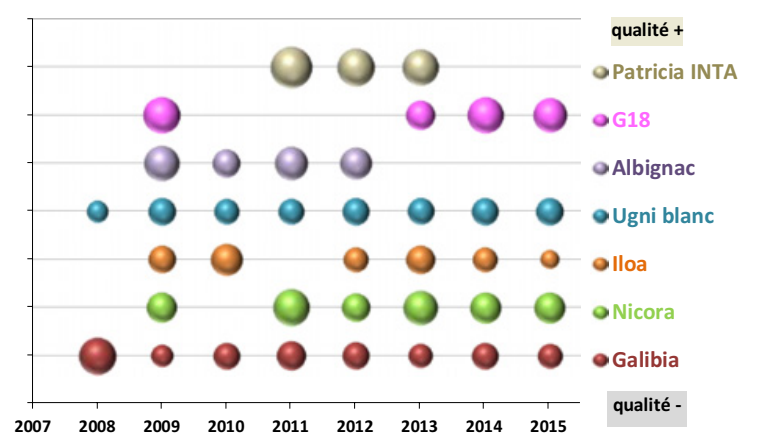

Figure 18. Evolution de la perception de l'intensité aromatique des jus blancs selon les millésimes et classification en fonction de la qualité. Grosseur de la boule en fonction de l'intensité des arômes en bouche.

polyphénol, les créations variétales INRA G3 et G4 ressortent favorablement pour leur potentiel aromatique.

\section{Conclusion}

Les conditions techniques et scientifiques ont été réunies pour que progressivement, en lien avec la société FOULON -SOPAGLY, une filière spécialisée puisse se mettre en place sur le continuum vigne-raisin-jus, de la vigne à la cave et au chai de conditionnement en bouteilles.

\section{Références}

[1] H. Ojeda, J.L. Escudier, G Albagnac, A. Sivry, P. Guyot. Diversification des produits de la vigne : création d'une filière jus de raisin. Revue des œnologues. $\mathbf{N}^{\circ}$ 130, 30-35 (2009)

[2] M. Mikolajczak, D. Bouissou, JL. Escudier, A. Sivry. Création d'une filière jus de raisin : Elaboration de jus de raisin aux caractéristiques maîtrisées. Revue des œnologues. $\mathbf{N}^{\circ} \mathbf{1 5 7}$, (oct. 2015)

[3] A. Samson, M. Veyret, S. Caillet, J.M. Souquet, V. Cheynier, A. Sivry. Création d'une filière jus de raisin : Evaluation qualitative physico-chimique et sensorielle des jus aux caractéritiques prédéfinies. Revue des œnologues. $\mathbf{N}^{\circ} \mathbf{1 5 8}$, (janvier 2016)

[4] H. Ojeda, J. Rousseau, E. Zumstein, M. Heywang, J.N. Lacapère, A. Sivry. Innovation variétale et étude agro,omique : Sélection des cépages et conduite du vignoble. Revue des œnologues. $\mathbf{N}^{\circ} \mathbf{1 5 8}$, (janvier 2016) 
[5] M. Mikolajczak, M. Veyret, P. Williams, T. Doco, JL. Escudier. Jus de raisin : Comment extraire le maximum du potentiel polyphènol de la baie de raisin. Revue française d'œnologie. $\mathbf{N}^{\circ} \mathbf{2 4 9}$, (2011)

[6] A. Bouquet. Du gène à la bouteille ! Quelles applications de l'étude des génomes en Viticulture

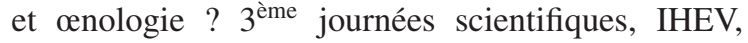
Montpellier Supagro, INRA. Pages 17 à 26, (2003), actes IHEV

[7] A. Bouquet, J. Pauquet, A.F. Adam-Blondon, L. Torregrosa, D. Merdinoglu, S.WiedemannMerdinoglu. Vers l'obtention de variétés de vigne résistantes à l'oïdium et au mildiou par les méthodes conventionnelles et biotechnologiques. Bull. OIV 833-834, 2000

[8] A. Feechan, M. Kocsis, S. Riaz, W. Zhang, D.M. Gadoury, M.A. Walker, I.B. Dry, B Reich, L. Cadle-Davidson. Genetics and resistance. Strategies for RUN 1 deployment using RUN2 and REN 2 to manage grape vine powdery mildew informed by studies of race specificity. Vol. 105.8, page 11041112. V (2015)

[9] Ojeda H., Mestre Sanchis F., Corbacho L., Bouquet A., Carbonneau A. 2010. Reduction of cost and emissions in vineyards by the use of lowinput technologies adapted to productions of good quality wines: genotypes resistant to fungal diseases and minimal pruning - zero pruning- system. Progrès Agricole et Viticole, $\mathbf{N}^{\circ}$ 21-22, 431-440 (2010)

[10] P. Duquene, A. Samson, C. Rapilly, B. Bernet, J.M. Salmon. Revue française d'oenologie. $\mathbf{N}^{\circ} \mathbf{2 6 6}, 8-16$ (oct. 2014)

[11] C .Rapimmy, G. Costagli, M. Franzoso, B. Bernet, R. Ferrarini. Revue des œnologues. $\mathbf{N}^{\circ} \mathbf{1 5 6}$, (2015)

[12] R. Ferrarini, G.M. Ciman, F. Lonardi, L. Bonetti, C. Rapilly, M. Franzoso, G. Costagli. $66^{\text {th }}$ ASEV National conference. Portland, Oregon, (juin 2015)

[13] J.L. Escudier, D. Bouissou, M Moutounet. Membrane based options to regulate $\mathrm{pH}$ and acidity. Proceedings of the 16th International enology symposium. (2011), Bolzano, Italy

[14] H. Ojeda, P. Rigal, M. Mikolajczak, A. Samson, B. Pagés, R. Schneider, G. Archambauld, S. Caillé, J.L. Escudier. Raisins verts : De la récolte à la transformation. Application à l'élaboration de verjus. (2008), PAV $\mathrm{N}^{\circ} 8$ 